PROCEEDINGS OF THE AMERICAN MATHEMATICAL SOCIETY

Volume 127, Number 3, March 1999, Pages 779-782

S $0002-9939(99) 04640-7$

\title{
ON THE PROOF OF CHARACTERIZATIONS OF THE EXPONENTIAL DICHOTOMY
}

\author{
NGUYEN VAN MINH
}

(Communicated by Palle E. T. Jorgensen)

\begin{abstract}
We describe explicitly the generator of the evolutionary semigroup associated with the evolutionary operator generated by the linear differential equation $x^{\prime}(t)=A(t) x(t)$. From this we give a short proof of some known characterizations of the exponential dichotomy of the above mentioned equation.
\end{abstract}

Consider the differential equations

$$
\begin{gathered}
u^{\prime}(t)=A(t) u(t), \\
u^{\prime}(t)=A(t) u(t)+f(t),
\end{gathered}
$$

where $A(t)$ are closed, generally unbounded linear operators on a Banach space $\mathbf{X}$, and $f: \mathbf{R} \rightarrow \mathbf{X}$ is a continuous function. Assuming well-posedness, Eq.(1) will generate an evolutionary operator $(U(t, s))_{t \geq s}$ consisting of bounded linear operators from $\mathbf{X}$ to itself with the following properties (see e.g. $[\mathrm{P}]$ ):

i) $U(t, t)=I, \forall t ;\|U(t, s)\| \leq N e^{\omega(t-s)}, \forall t \geq s$,

ii) $U(t, r) U(r, s)=U(t, s)(t \geq r \geq s)$,

iii) the map $(t, s) \mapsto U(t, s) x$ is continuous for every fixed $x(t \geq s)$.

In this paper, instead of Eq.(1), we shall deal with families of operators with the above mentioned properties, called evolutionary families, generated by them. One of the central topics of the qualitative theory of differential equations is to find conditions for Eq.(1) to admit exponential dichotomy. We recall that Eq.(1) is said to admit exponential dichotomy (see e.g. [D-K], $[\mathrm{L}-\mathrm{M}]$ ) if there exists a family of projections $P(t), t \in \mathbf{R}$, and positive constants $M, \alpha$ such that

i) for every fixed $x \in \mathbf{X}$, the map $t \mapsto P(t) x$ is continuous,

ii) $P(t) U(t, s)=U(t, s) P(s), \forall t \geq s$,

iii) $\|U(t, s) x\| \leq M e^{-\alpha(t-s)}\|x\|, \forall t \geq s, x \in \operatorname{ImP}(s)$,

iv) $\|U(t, s) y\| \geq M^{-1} e^{\alpha(t-s)}\|y\|, \forall t \geq s, y \in \operatorname{KerP}(s)$,

v) $\left.U(t, s)\right|_{K \operatorname{erP}(s)}$ is an isomorphism from $\operatorname{KerP}(s)$ onto $\operatorname{KerP}(t), \forall t \geq s$.

Received by the editors December 20, 1996 and, in revised form, June 20, 1997.

1991 Mathematics Subject Classification. Primary 34G10, 47D06; Secondary 47H20.

Key words and phrases. Evolutionary family, evolutionary semigroup, exponential dichotomy.

This note was written during the author's visit to the Institute of Mathematics, University of Tübingen. The support of the Alexander von Humboldt Foundation is gratefully acknowledged. He also thanks R. Nagel and the Division of Functional Analysis at the Institute for their warm hospitality and constant encouragement. Finally, he thanks the referee for the valuable suggestion to improve the presentation of this paper.

(C)1999 American Mathematical Society 
It is the classical theorem of Perron that Eq.(1) admits exponential dichotomy if and only if for every bounded continuous $f$, there is a unique bounded solution on the real line to Eq.(2) (see e.g. [D-K, Chapter 4]). Zhikov [Zh] extended Perron's result to Eq.(1) in general Banach spaces with unbounded coefficients. He proved that Eq.(1) admits exponential dichotomy if and only if the differential operator $L=d / d t-A(t)$ is invertible (or regular, in the terminology of [Zh]).

Recently, there has been increasing interest in the asymptotic behaviour of Eq.(1) with the approach based on the so-called evolutionary semigroups associated with evolutionary families (see e.g. [R], $[\mathrm{M}],[\mathrm{L}-\mathrm{M}]$ ). Recall that the evolutionary semigroup $\left(T^{h}\right)_{h \geq 0}$ associated with $(U(t, s))_{t \geq s}$ is a $C_{0}$-semigroup on $C_{0}(\mathbf{R}, \mathbf{X})=$ $\left\{v: \mathbf{R} \rightarrow \mathbf{X}\right.$ continuous, $\left.\lim _{t \rightarrow \infty}\|v(t)\|=0\right\}$ defined by

$$
\left[T^{h} v\right](t)=U(t, t-h) v(t-h), \forall h \geq 0, t \in \mathbf{R}, v \in C_{0}(\mathbf{R}, \mathbf{X})
$$

(See e.g. [L-M], [R].) We shall denote the generator of $\left(T^{h}\right)_{h \geq 0}$ by $\mathcal{A}$. In $[\mathrm{L}-\mathrm{M}]$, it was proved that, for separable Banach spaces $X$, the evolutionary family $(U(t, s))_{t \geq s}$ admits exponential dichotomy if and only if $\mathcal{A}$ is invertible, or equivalently, if and only if the spectrum of $T^{h}$ has empty intersection with the unit circle (for some, and hence for all $h>0$ ). This result can also be reformulated as a spectral mapping theorem for the evolutionary semigroup (see [L-M] and Corollaries 1, 3 below).

It turns out that the generator $\mathcal{A}$ is in fact the differential operator $L$ used by Zhikov in $C_{0}(\mathbf{R}, \mathbf{X})$ defined by the equation

$$
x(t)=U(t, s) x(s)+\int_{s}^{t} U(t, \xi) f(\xi) d \xi \quad(\forall t \geq s) .
$$

In connection with the unique solvability of (3) the quoted result of Zhikov [Zh, Lemma 1] can be reformulated as follows: a given evolutionary family $(U(t, s))_{t \geq s}$ has an exponential dichotomy if and only if for every bounded and continuous function $f, E q$. (3) has a unique bounded and continuous solution on the real line.

The purpose of this paper is to show how Zhikov's result and the precise description of the generator $\mathcal{A}$ imply Latushkin-Montgomery-Smith's result, even without the separability assumption on $\mathbf{X}$. In fact, we observe that the generator $\mathcal{A}$ of the evolutionary semigroup $\left(T^{h}\right)_{h \geq 0}$ can be defined (implicitly) through Eq.(3) (see Lemma 1 below), which allows us to derive the result of Latushkin and Montgomery-Smith immediately from that of Zhikov.

The main point in our analysis is to consider the affine semigroup $\left(T_{f}^{h}\right)_{h \geq 0}$ associated with Eq.(3) for $f \in C_{0}(\mathbf{R}, \mathbf{X})$, defined as follows:

$$
T_{f}^{h} v=T^{h} v+\int_{0}^{h} T^{h-\xi} f d \xi=T^{h} v+\int_{0}^{h} T^{\xi} f d \xi
$$

where $v \in C_{0}(\mathbf{R}, \mathbf{X}), h \geq 0$ (for more information on $\left(T^{h}\right)_{h \geq 0}$ in the general case see $[\mathrm{A}-\mathrm{M}])$. It is easily checked that for all $v \in C_{0}(\mathbf{R}, \mathbf{X})$ we have

$$
\left[T_{f}^{h} v\right](t)=U_{f}(t, t-h) v(t-h), t \in \mathbf{R}, h \geq 0,
$$

where $U_{f}(t, s)$ is the evolutionary operator defined by the integral equation (3).

Lemma 1. The action of the generator $\mathcal{A}$ can be described as follows: $g \in D(\mathcal{A})$ if and only if there exists $f \in C_{0}(\mathbf{R}, \mathbf{X})$ such that $\mathcal{A} g=-f$ and

$$
g(t)=U(t, s) g(s)+\int_{s}^{t} U(t, \xi) f(\xi) d \xi \quad(\forall t \geq s) .
$$


Proof. The lemma can easily be proved by observing that the assertion that $g, f \in$ $C_{0}(\mathbf{R}, \mathbf{X})$ and $g$ is a solution of (3), is equivalent to $T_{f}^{h} g=g(\forall h \geq 0)$. From (4) this is equivalent to

$$
\begin{gathered}
g=T_{f}^{h} g=T^{h} g+\int_{0}^{h} T^{\xi} f d \xi=T_{f}^{h} g, \forall h \geq 0, \\
T^{h} g-g=\int_{0}^{h} T^{\xi} A g d \xi=-\int_{0}^{h} T^{\xi} f d \xi \forall h \geq 0 .
\end{gathered}
$$

From the general theory of linear operator semigroups $[\mathrm{P}, \mathrm{p} .4-5]$ this is equivalent to the assertion $\mathcal{A} g=-f$.

Now we state the $C_{0}(\mathbf{R}, \mathbf{X})$ version of Zhikov's result which can be proved in the same way as in [L-Z, chap. 10].

Theorem 1. The family $(U(t, s))_{t \geq s}$ has an exponential dichotomy if and only if for every $f \in C_{0}(\mathbf{R}, \mathbf{X})$ there exists a unique $g \in C_{0}(\mathbf{R}, \mathbf{X})$ which solves (3).

The following Corollaries 1, 2 are direct consequences of Lemma 1 and Theorem 1.

Corollary 1. The family $(U(t, s))_{t \geq s}$ has an exponential dichotomy if and only if $\mathcal{A}$ is invertible.

Corollary 2. If $\lambda \in \sigma(\mathcal{A})$, then $\lambda+i \mu \in \sigma(\mathcal{A}) \forall \mu \in \mathbf{R}$.

Corollary 3. The evolutionary family $(U(t, s))_{t \geq s}$ has an exponential dichotomy if and only if $T^{h}$ is hyperbolic for some (and thus for all) $h>0$.

Proof. Since the "only if" part is trivial, we need only prove the "if" part. Suppose to the contrary that the family $(U(t, s))_{t \geq s}$ doesn't admit exponential dichotomy. It follows from Corollary 1 that $0 \in \sigma(\mathcal{A})$. By the Spectral Inclusion Theorem, we have $e^{h \sigma(\mathcal{A})} \subset \sigma\left(T^{h}\right)$ (see $\left.[\mathrm{P}]\right)$ and hence, by Corollary 2, the unit circle is contained in $\sigma\left(T^{h}\right)$, which is a contradiction.

Remark 1 . The $L_{p^{-}}$-version of the above mentioned proofs can be done in the same way.

\section{REFERENCES}

[A-M] Aulbach B., Nguyen Van Minh, Nonlinear semigroups and the existence and stability of solutions of semilinear nonautonomous evolution equations, Abstract and Applied Analysis 1 (1996), 351-380.

[D-K] Daleckii Ju. L., Krein M.G., "Stability of Solutions of Differential Equations in Banach Space", Amer. Math. Soc., Providence RI, 1974. MR 50:5126

[L-M] Latushkin Ju., Montgomery-Smith S., Evolutionary semigroups and Lyapunov theorems in Banach spaces J. Func. Anal. 127(1995), 173-197. MR 96k:47022

[L-Z] Levitan B.M., Zhikov V.V., "Almost Periodic Functions and Differential Equations", Moscow Univ. Publ. House 1978. English translation by Cambridge University Press 1982. MR 84g:34004

[M] Nguyen Van Minh, Semigroups and stability of nonautonomous differential equations in Banach spaces, Trans. Amer. Math. Soc., 345(1994), N.1, 223-242. MR 95a:34091

[P] Pazy A., "Semigroups of Linear Operators and Applications to Partial Differential Equations", Applied Math. Sci. 44, Springer-Verlag, Berlin-New York 1983. MR 85g:47061 
[R] Rau R., Hyperbolic evolutionary semigroups on vector valued function spaces, Semigroup Forum 48(1994), 107-118.

[Zh] Zhikov V.V., On the theory of admissibility of pairs of function spaces, Soviet Math. Dokl. vol. 13(1972), N.4, 1108-1111.

Department of Mathematics, The University of Electro-Communications, 1-5-1 ChofuGAOKA, ChOFu, TOKYo 182, JAPAN

E-mail address: minh@matha.e-one.uec.ac.jp 